\title{
FULL-WAVE ANALYSIS AND DESIGN OF BROADBAND TURNSTILE JUNCTIONS
}

\author{
A. A. San Blas ${ }^{1,}{ }^{*}$, F. J. Pérez ${ }^{2}$, J. Gil ${ }^{2}$, F. Mira ${ }^{3}$, \\ V. E. Boria ${ }^{4}$, and B. Gimeno ${ }^{5}$
}

${ }^{1}$ Departamento de Ingeniería de Comunicaciones, Universidad Miguel Hernández de Elche, Avda. de la Universidad s/n, E-03202 Elche, Alicante, Spain

${ }^{2}$ Aurora Software and Testing, S.L., Camino de Vera s/n, E-46022 Valencia, Spain

${ }^{3}$ Centre Tecnològic de Telecomunicacions de Catalunya, Avda. del Canal Olímpic s/n, E-08860 Castelldefels, Barcelona, Spain

${ }^{4}$ Departamento de Comunicaciones, iTEAM, Universidad Politécnica de Valencia, Camino de Vera s/n, E-46022 Valencia, Spain

${ }^{5}$ Departamento de Física Aplicada y Electromagnetismo - I.C.M.U.V., Universidad de Valencia, Dr. Moliner, 50, E-46100 Burjasot, Valencia, Spain

Abstract - The efficient design of broadband turnstile junctions compensated with piled-up cylindrical metallic posts is discussed in this contribution. Very high relative bandwidths can be obtained, while maintaining the diameter of the input circular access port lower than the width of the rectangular waveguide ports, thus reducing the number of excited higher order circular waveguide modes. A novel full-wave analysis tool has also been implemented in order to reduce the CPU effort related to the complete design process.

\section{INTRODUCTION}

A turnstile junction is a five-port device widely used in high-capacity telecommunication systems. This microwave and millimeter-wave component aims at separating the two orthogonal polarizations (horizontal and vertical) associated to the fundamental mode $\mathrm{TE}_{11}$

Received 17 May 2011, Accepted 2 June 2011, Scheduled 10 June 2011

* Corresponding author: A. A. San Blas (aasanblas@umh.es). 


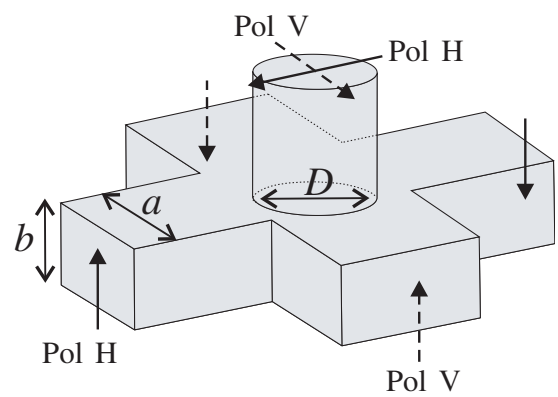

Figure 1. Turnstile junction. The dimensions of the output rectangular waveguide ports are $a \times b$, and the diameter of the input circular waveguide port is $D$.

of the input circular waveguide port of the device, as depicted in Fig. 1. This microwave network is a dominant component in current orthomode transducers (OMTs) which are present in a great variety of practical applications, such as dual-polarized antenna feed systems, remote-sensing radiometers and radio astronomy receivers [14]. Consequently, recent years have witnessed active research efforts focused on increasing the operation bandwidth of turnstile junctions. To reach this goal, several solutions have been proposed in the technical literature in the past few years, thus giving rise to the so-called compensated turnstile junctions. Different compensation techniques have also been applied recently to the optimization of other multiport waveguide junctions, such as crossed waveguide junctions, T-junctions, right-angled bends and magic tees $[5,6]$.

With regard to the compensation of turnstile junctions, an OMT was designed in [1] by compensating the turnstile junction using a double-cylindrical post, and the electrical performance of the turnsile junction presented in [2] was optimized by adding a square prism located at the base of the structure. More recently, a metallic pyramid tuning stub was used in [3], and a single cylindrical metallic post was proposed in [7] as a compensating element. Although the turnstile junctions designed in the contributions $[1-3]$ can achieve a high relative bandwidth, it is important to note that the diameter $D$ of the input circular waveguide port used in these structures is greater than the width $a$ of the output rectangular access ports. For example, we have that $D=1.1933 \cdot a$ in $[1] ; D=1.084 \cdot a$ in [2]; and a diameter $D=1.1374 \cdot a$ in [3]. The use of a circular waveguide whose diameter satisfies $D>a$ has the following drawbacks:

- As the diameter $D$ of the circular waveguide becomes greater, the cutoff frequency of higher order modes decreases. Therefore, 
although their excitation can be avoided as long as the symmetry of the turnstile junction is kept, the fabrication process can introduce undesired mechanical defects which can lead to the excitation of such other modes, thus affecting to the electrical performance of the device.

- The structure cannot be analyzed using standard modal methods, so it is necessary to resort to numerical techniques in order to rigorously characterize the device. As a consequence, the computational cost of the design process is dramatically increased.

In that respect, it is important to point up that the turnstile junctions presented in [1-3] have been analyzed and designed using commercial tools based on finite-element or finite-integration methods. Consequently, we might expect a huge CPU effort in order to optimize the electrical performance of the device, since the tuning stub used in the previous contributions has, at least, two optimizable dimensions (four in the case of [1]). Moreover, apart from the fundamental mode $\mathrm{TE}_{11}$ of the circular waveguide, other two higher order modes (i.e., $\mathrm{TM}_{01}$ and $\mathrm{TE}_{21}$ ) can be excited in the frequency range of the operation band of the turnstile junctions designed in [1-3]. Therefore, the fabrication process must be accurate enough in order to preserve the symmetry of the component, thus avoiding the potential excitation of these higher order circular waveguide modes.

In this work, we present a novel Computer-Aided Design (CAD) tool for the rigorous modal analysis and design of compensated turnstile junctions. The implemented modal technique, which is based on the 3-D Boundary Integral-Resonant Mode Expansion method [8], is very efficient from a computational point of view, thus minimizing the CPU effort related to the design process with respect to previous contributions. In addition to this, the diameter $D$ of the circular waveguide of the designed turnstile junctions satisfies $D \leq a$ with the aim of reducing the number of excited higher order modes. Therefore, the main goal of this contribution is to demonstrate that it is possible to design broadband turnstile junctions with a very high relative bandwidth (up to $42.3 \%$ ), while maintaining $D \leq a$ in order to increase the cutoff frequency of higher order modes.

The tuning stub used to compensate the structure consists of five piled-up cylindrical metallic posts (see Fig. 2) placed on the base of the junction in a centered position in order to preserve the symmetry of the component. It is important to notice that the post situated at the top of the tuning stub is introduced into the region of the circular waveguide for the purpose of improving the electrical response of the device, while adding a new degree of freedom to the design process. 


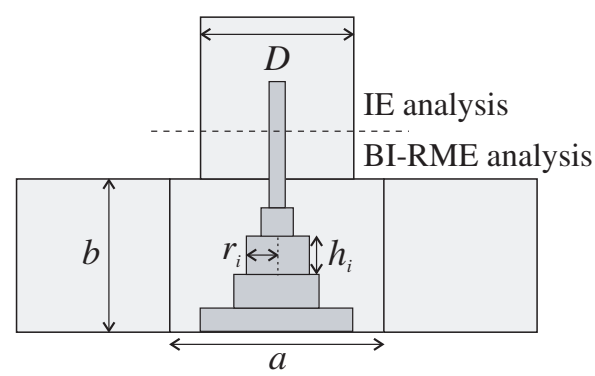

Figure 2. Compensated turnstile junction using 5 piled-up cylindrical posts. The radius and height of each post are $r_{i}$ and $h_{i}$, respectively, with $i=1,2, \ldots, 5$.

\section{FULL-WAVE ANALYSIS OF COMPENSATED TURNSTILE JUNCTIONS}

The full-wave analysis of the compensated turnstile junction represented in Fig. 2 is divided into two main stages. First of all, an integral equation (IE) technique [9] is used to obtain a generalized impedance matrix (GIM) representation of the planar junction between the circular waveguide and the coaxial waveguide that we find in the turnstile junction (see Fig. 2). The GIM of the planar junction can be written in the form:

$$
Z_{m, n}^{(\xi, \gamma)}=\int_{S^{(2)}} \mathbf{M}_{n}^{(\gamma)}(x, y) \cdot \mathbf{h}_{m}^{(\xi) *}(x, y) d S
$$

where $(\xi),(\gamma)=(1),(2)$ represent the two waveguide ports involved in the planar junction; $S^{(2)}$ is the cross section of the coaxial waveguide; $\mathbf{h}_{m}^{(\xi)}(x, y)$ is the $m$-th magnetic-type vector modal function of the waveguide port $(\xi)$; and $\mathbf{M}_{n}^{(\gamma)}(x, y)$ are unknown vector functions that can be expanded in terms of the magnetic-type vector modal functions of the waveguide port $(\gamma)$ [9]. Moreover, an alternative formulation based on the method of moments has been followed in order to obtain the modal chart of the circular and coaxial waveguides [10].

In order to validate the software tool related to this stage, we analyze a planar junction between a circular waveguide $(D=$ $13.98 \mathrm{~mm}$ ) and a coaxial waveguide whose external diameter is equal to $D$ and the inner diameter is $3.0 \mathrm{~mm}$. The magnitude of the reflection coefficient is presented in Fig. 3 and the obtained results are successfully compared with data provided by a commercial finiteelement method (FEM) tool ${ }^{\dagger}$. In this case, the excitation mode is the

$\dagger$ HFSS rel. 11.1.3, Ansoft Corporation, Pittsburgh, PA, 2008. 
fundamental mode $\mathrm{TE}_{11}$ of the circular waveguide and the response mode is the $\mathrm{TE}_{11}$ mode of the coaxial waveguide.

Afterwards, the 3-D Boundary Integral-Resonant Mode Expansion technique (BI-RME) [8] is employed to derive the generalized admittance matrix (GAM) of the rest of the structure, that is, a component with four rectangular waveguide ports and a coaxial access port (see Fig. 2). To this end, the authors have properly extended the theoretical work developed in [11], thus allowing to consider an increased number of access ports. The expression of the obtained GAM is:

$$
\mathbf{Y}=\frac{1}{j k \eta} \mathbf{Y}^{\mathrm{A}}+\frac{j k}{\eta} \mathbf{Y}^{\mathrm{B}}+\frac{j k^{3}}{\eta} \mathbf{Y}^{\mathrm{C}}, \quad k \leq 2 \pi f_{\max \sqrt{\mu \epsilon}}
$$

where $k$ is the wavenumber, $\eta$ represents the wave impedance, and the expressions of the matrices $\mathbf{Y}^{\mathrm{A}}, \mathbf{Y}^{\mathrm{B}}$ and $\mathbf{Y}^{\mathrm{C}}$ can be found in [8]. Matrices $\mathbf{Y}^{\mathrm{A}}$ and $\mathbf{Y}^{\mathrm{B}}$ are frequency-independent, whereas the matrix $\mathbf{Y}^{\mathrm{C}}$ is frequency-dependent. Moreover, in order to compute the elements of the matrices $\mathbf{Y}^{\mathrm{A}}$ and $\mathbf{Y}^{\mathrm{B}}$, the following set of matrices related to the waveguide access ports has to be calculated:

$$
\begin{aligned}
G_{\ln } & =\int_{S} \int_{S^{\prime}} \nabla_{S} \cdot \mathbf{h}_{l}(\mathbf{r}) g^{m}\left(\mathbf{r}, \mathbf{r}^{\prime}\right) \nabla_{S}^{\prime} \cdot \mathbf{h}_{n}\left(\mathbf{r}^{\prime}\right) d S d S^{\prime} \\
T_{\ln } & =\int_{S} \int_{S^{\prime}} \mathbf{h}_{l}(\mathbf{r}) \cdot \overrightarrow{\mathbf{G}}_{0}^{\mathrm{F}}\left(\mathbf{r}, \mathbf{r}^{\prime}\right) \cdot \mathbf{h}_{n}\left(\mathbf{r}^{\prime}\right) d S d S^{\prime} \\
L_{\mathrm{ln}} & =\int_{S} \int_{S^{\prime}} \mathbf{N}_{l}(\mathbf{r}) \cdot \nabla \times \overrightarrow{\mathbf{G}}_{0}^{\mathrm{F}}\left(\mathbf{r}, \mathbf{r}^{\prime}\right) \cdot \mathbf{h}_{n}\left(\mathbf{r}^{\prime}\right) d S d S^{\prime} \\
F_{\ln } & =\int_{S} \mathbf{H}_{l}(\mathbf{r}) \cdot \mathbf{h}_{n}(\mathbf{r}) d S .
\end{aligned}
$$

In the previous expressions, $g^{\mathrm{m}}\left(\mathbf{r}, \mathbf{r}^{\prime}\right)$ and $\overrightarrow{\mathbf{G}}_{0}^{\mathrm{F}}\left(\mathbf{r}, \mathbf{r}^{\prime}\right)$ are the quasi-static magnetic-type Green's functions related to a rectangular resonator whose dimensions are $a \times b \times a ; \mathbf{h}_{n}(\mathbf{r})$ is the $n$-th magnetic-type vector modal function of a waveguide port (in our case, circular or rectangular waveguide ports); $\mathbf{N}_{l}(\mathbf{r})$ is the $l$-th base function used to describe the electric current over the tuning stub; and $\mathbf{H}_{l}(\mathbf{r})$ is the $l$-th magnetictype resonant mode of the rectangular resonator $a \times b \times a$. After computing the elements of the previous matrices, the GAM of the structure can be readily obtained using expression (2).

Finally, the obtained GIM and GAM are conveniently connected in order to yield a full-wave representation of the whole component. The efficiency of the methods implemented makes this software tool very suitable to optimize the electromagnetic response of the components designed. 


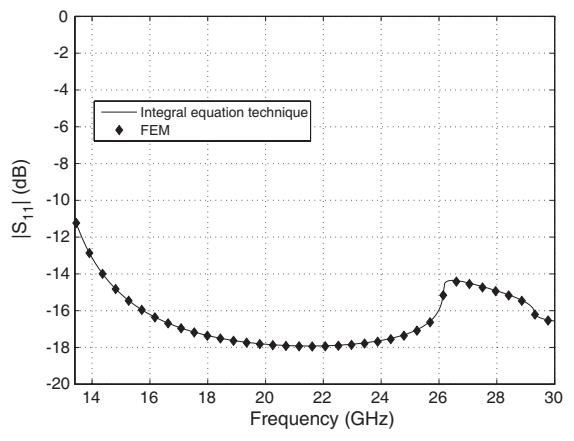

Figure 3. Reflection coefficient of a planar junction between a circular waveguide and a coaxial waveguide.

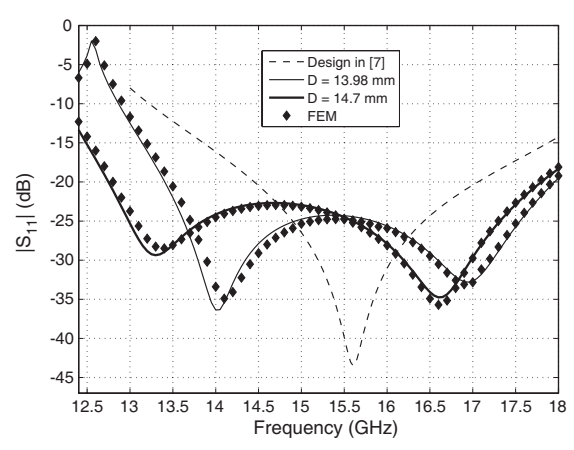

Figure 4. Design of a compensated turnstile junction using WR-62 rectangular waveguide ports.

\section{DESIGN OF BROADBAND COMPENSATED TURNSTILE JUNCTIONS}

Firstly, we focus our attention on the validation of the developed CAD tool. To this aim, we start from a design presented in a previous authors' work with the objective of improving the relative bandwidth of the compensated turnstile junction. In the design presented in [7], the rectangular waveguide ports are WR-62 standard waveguides ( $a=15.8 \mathrm{~mm}, b=7.9 \mathrm{~mm}$ ), the diameter of the circular waveguide is $D=13.98 \mathrm{~mm}$ (note that $D<a$ ) and the relative bandwidth below $-20 \mathrm{~dB}$ is about $16.5 \%$.

We can optimize the electrical performance of the turnstile junction by introducing a compensating tuning stub composed of five piled-up cylindrical posts, as represented in Fig. 2. The optimal radii and heights of the piled-up cylindrical posts can be found in Table 1, where it is assumed that the posts are ordered from bottom to top. The magnitude of the reflection coefficient (the excitation mode is the fundamental mode of the circular waveguide) of the new designed turnstile junction is shown in Fig. $4(D=13.98 \mathrm{~mm})$, and our results are successfully compared with data provided by the commercial finite-element method (FEM) tool. The relative bandwidth below $-20 \mathrm{~dB}$ of this new design is about $26 \%$.

In the same figure, the results presented in [7] have been included, and we have also added new results for the case with $D=14.7 \mathrm{~mm}$ (the dimensions of the tuning stub in this case are very similar to the ones described in Table 1). We observe that the new relative bandwidth in this last design is about $33.1 \%$. Moreover, since $D<a$ in both new 
designs, only one higher order mode of the circular waveguide $\left(\mathrm{TM}_{01}\right)$ can be excited in the operation frequency band of the device (i.e., above $15.61 \mathrm{GHz}$ ). Finally, it should be emphasized that the complete simulation of the component just took 1.9 seconds per frequency point on a PC with $4 \mathrm{~GB}$ of RAM. On the other hand, the commercial finite-element method-based tool consumed around 20 minutes of $\mathrm{CPU}$ time.

Next, we present a new design using the same rectangular waveguide ports as the ones described in [2]. In this case, $a=10.7 \mathrm{~mm}$, $b=5.3 \mathrm{~mm}$ and $D=a$ (note that a diameter $D=11.6 \mathrm{~mm}$ is used in [2]). The dimensions of the piled-up posts used in this new design are presented in Table 2.

The magnitude of the obtained reflection coefficient is represented in Fig. 5, where an excellent agreement with the results provided by the finite-element method software is observed. The relative bandwidth below $-20 \mathrm{~dB}$ obtained in this design is $42.3 \%$, and this value is very close to the relative bandwidth achieved in the design presented in [2], which is about $44.8 \%$. The main advantage of this new design with respect to the one presented in [2] lies in the fact that we have reduced the radius of the circular waveguide port, thus avoiding the potential excitation of the second higher order mode $\mathrm{TE}_{21}$, whose cutoff frequency $(27.24 \mathrm{GHz})$ is out of the frequency range of the operation band $(18-26 \mathrm{GHz})$ of the OMT designed in [2]. On the contrary, the cutoff frequency of the second higher order mode $\mathrm{TE}_{21}$ in the design presented in [2] is $25.13 \mathrm{GHz}$, and this value is, effectively, in the operation band of the designed OMT. Therefore, our new design reduces the number of higher order modes that can be potentially

Table 1. Design 1. Radii and heights of the piled-up cylindrical posts used in the design $(D=13.98 \mathrm{~mm})$ presented in Fig. 4 .

\begin{tabular}{|c|c|c|c|c|c|}
\hline \hline$r_{i}(\mathrm{~mm})$ & 4.45 & 2.5 & 1.4 & 1.1 & 0.9 \\
\hline$h_{i}(\mathrm{~mm})$ & 1.9 & 1.6 & 1.9 & 1.3 & 10.2 \\
\hline \hline
\end{tabular}

Table 2. Design 2. Radii and heights of the piled-up cylindrical posts used in the design presented in Fig. 5.

\begin{tabular}{|c|c|c|c|c|c|}
\hline \hline$r_{i}(\mathrm{~mm})$ & 3.75 & 1.7 & 1.2 & 0.9 & 0.6 \\
\hline$h_{i}(\mathrm{~mm})$ & 1.2 & 1.1 & 1.3 & 0.9 & 7.6 \\
\hline \hline
\end{tabular}


excited due to undesired defects during the fabrication process, while maintaining a similar relative bandwidth. In addition to this, the implemented simulation tool is very efficient from a computational point of view, thus dramatically reducing the CPU effort devoted to the complete design process.

Finally, we present a compensated turnstile junction that, as the one described in [1], is implemented in WR-10 rectangular waveguide $(a=2.54 \mathrm{~mm}, b=1.27 \mathrm{~mm})$. In this new design, the diameter of the circular waveguide is $D=2.52 \mathrm{~mm}$, whereas the diameter used in [1] is greater than $a(D=3.031 \mathrm{~mm})$. The dimensions of the compensating tuning stub are presented in Table 3 , and the reflection coefficient of the compensated turnstile junction is shown in Fig. 6. As we can observe, the relative bandwidth below $-20 \mathrm{~dB}$ is about $37.8 \%$, which is equal to the one achieved in [1], thus demonstrating that it is possible to optimize the electrical response of the device maintaining $D<a$. In this alternative design, the cutoff frequency of the $\mathrm{TE}_{21}$ mode is increased up to $115.66 \mathrm{GHz}$, thus avoiding its potential excitation (the cutoff frequency of the $\mathrm{TE}_{21}$ mode is $96.16 \mathrm{GHz}$ in [1]).

Table 3. Design 3. Radii and heights of the piled-up cylindrical posts used in the design presented in Fig. 6.

\begin{tabular}{|c|c|c|c|c|c|}
\hline \hline$r_{i}(\mathrm{~mm})$ & 0.8 & 0.4 & 0.23 & 0.18 & 0.15 \\
\hline$h_{i}(\mathrm{~mm})$ & 0.3 & 0.27 & 0.3 & 0.2 & 1.8 \\
\hline \hline
\end{tabular}

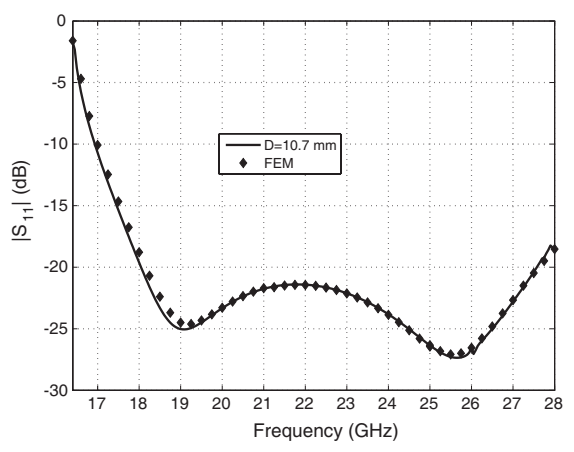

Figure 5. Design of a compensated turnstile junction using rectangular waveguide ports with $a=10.7 \mathrm{~mm}$ and $b=5.3 \mathrm{~mm}$.

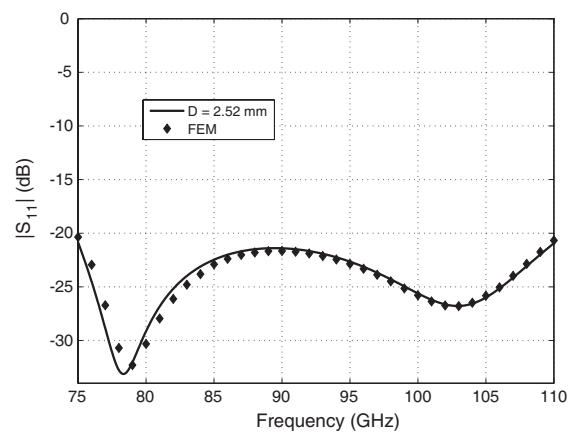

Figure 6. Design of a compensated turnstile junction using standard WR-10 rectangular waveguide ports. 


\section{CONCLUSION}

In this contribution, an efficient full-wave CAD tool for the analysis and design of broadband turnstile junctions compensated with piledup cylindrical posts has been discussed. Three different designs with a high relative bandwidth similar to the obtained in recently published works have been presented. The diameter of the circular waveguides used in the different designs is not greater than the width of the rectangular waveguide ports, thus avoiding the potential excitation of higher order circular waveguide modes due to undesired mechanical defects that may occur during the fabrication process.

\section{REFERENCES}

1. Pisano, G., L. Pietranera, K. Isaak, L. Piccirillo, B. Johnson, B. Maffei, and S. Melhuish, "A broadband WR10 turnstile junction orthomode transducer," IEEE Microw. Wirel. Compon. Lett., Vol. 17, No. 4, 286-288, 2007.

2. Navarrini, A. and R. L. Plambeck, "A turnstile junction waveguide orthomode transducer," IEEE Trans. Microw. Theory Tech., Vol. 54, No. 1, 272-277, 2006.

3. Park, S., H. Lee, and Y. Kim, "A turnstile junction waveguide orthomode transducer for the simultaneus dual polarization radar," Asia-Pacific Microw. Conf., 135-138, 2009.

4. Peverini, O. A., R. Tascone, G. Virone, A. Olivieri, and R. Orta, "Orthomode transducer for millimeter-wave correlation receivers," IEEE Trans. Microw. Theory Tech., Vol. 54, No. 5, 2042-2049, 2006.

5. El Sabbagh, M. and K. Zaki, "Modeling of rectangular waveguide junctions containing cylindrical posts," Progress In Electromagnetics Research, Vol. 33, 299-331, 2001.

6. Wu, H. C. and W. B. Dou, "A rigorous analysis and experimental researches of waveguide magic tee at W band," Progress In Electromagnetics Research, Vol. 60, 131-142, 2006.

7. San Blas, A. A., F. Mira, J. Gil, V. E. Boria, and B. Gimeno, "Efficient analysis and design of compensated turnstile junctions using advanced modal techniques," Progress In Electromagnetics Research Letters, Vol. 12, 21-30, 2009.

8. Arcioni, P., M. Bozzi, M. Bressan, G. Conciauro, and L. Perregrini, "Frequency/time-domain modeling of 3-D waveguide structures by a BI-RME approach," Int. J. Numer. Model.-Electron. Netw. Device Fields, Vol. 15, 3-21, 2002. 
9. Gerini, G., M. Guglielmi, and G. Lastoria, "Efficient integral equation formulations for admittance or impedance representation of planar waveguide junctions," IEEE MTT-S Int. Dig., Vol. 3, 1747-1750, 1998.

10. Gimeno, B. and M. Guglielmi, "Multimode equivalent network representation for junctions between coaxial and circular waveguides," Int. J. Microw. Millimet.-Wave Comput.-Aided Eng., Vol. 7, No. 2, 180-194, 1997.

11. San Blas, A. A., F. Mira, V. E. Boria, B. Gimeno, G. Conciauro, M. Bressan, and P. Arcioni, "Efficient CAD of generalized coaxial probes in rectangular waveguide using the 3D BI-RME method," 36th European Microw. Conf., 1163-1166, 2006. 